\title{
The General Relativistic Hydrogen Atom
}

\author{
Jeffrey M. Cohen ${ }^{1 \star}$ and Robert T. Powers ${ }^{2 \star}$ \\ 1 Department of Physics, University of Pennsylvania, Philadelphia, PA 19104, USA \\ 2 Mathematics Department, University of Pennsylvania, Philadelphia, PA 19104, USA
}

\begin{abstract}
The general relativistic Dirac equation is formulated in an arbitrary curved space-time using differential forms. These equations are applied to spherically symmetric systems with arbitrary charge and mass. For the case of a black hole (with event horizon) it is shown that the Dirac Hamiltonian is selfadjoint, has essential spectrum the whole real line and no bound states. Although rigorous results are obtained only for a spherically symmetric system, it is argued that, in the presence of any event horizon there will be no bound states. The case of a naked singularity is investigated with the results that the Dirac Hamiltonian is not self-adjoint. The self-adjoint extensions preserving angular momentum are studied and their spectrum is found to consist of an essential spectrum corresponding to that of a free electron plus eigenvalues in the gap $\left(-m c^{2}\right.$, $\left.+m c^{2}\right)$. It is shown that, for certain boundary conditions, neutrino bound states exist.
\end{abstract}

\section{Introduction}

In this paper we formulate the Dirac equation in an arbitrary curved space-time (Sect. I). These equations are applied to spherically symmetric systems with arbitrary charge and mass (Sect. II and III). For the case of a black hole (with an event horizon) we show that the Dirac Hamiltonian is self-adjoint, has essential spectrum the whole real line and no bound states (no point spectrum) (Sect. IV). Although rigorous results are only obtained for the case of spherical symmetry, it is argued that in the presence of any event horizon there will be no bound states.

In Sect. $\mathrm{V}$ the case of a naked singularity is investigated. For a proton or any atom in the periodic table, the charge to mass ratio is such that the corresponding Riesner-Nordstrom solution has a naked singularity. For the case of a naked singularity we show the Dirac Hamiltonian is not self-adjoint. The deficiency indices are $(\infty, \infty)$ but for each angular momentum subspace $\mathfrak{M}_{k m}(k= \pm 1, \pm 2, \pm$ $3, \ldots, m= \pm 1 / 2, \pm 3 / 2, \ldots$ ) labelled by two quantum numbers $k$ and $m$, the

* Supported in part by the National Science Foundation 
Hamiltonian restricted to $\mathfrak{M}_{k m}$ has deficiency indices $(1,1)$. The self-adjoint extensions preserving these subspaces are studied. The extended Hamiltonian is labeled by a parameter $\theta$ with $0<\theta<\pi$ corresponding to a boundary condition imposed at the origin. The extended Hamiltonian $H_{\theta}$ has essential spectrum $(-\infty,-m] \cup[m, \infty)$ and all bound states are confined to the interval $[-m, m]$ (i.e. no bound states in the continuum.) In general there are bound states. What is surprising is that for certain values of $\theta$ between $\pi / 2$ and $\pi$ there appears a "new" bound state. For example, rough calculations indicated that with a proper choice of $\theta$, one will have a hydrogen atom with a state of energy $\lambda$ anywhere in the interval $(-m, m)$. In particular one could have a new ground state.

\section{Curved Space Dirac Equation}

An important innovation in differential geometry is E. Cartan's calculus of differential forms and movable frames $[1,6]$. Familiar to mathematicians since 1901, differential forms have recently been applied to physical problems. It is particularly appropriate to express Maxwell's equations [3] for electromagnetism and Einstein's equations for gravity. It has been shown $[1,6]$ that the curved space Dirac equation for spin- $\frac{1}{2}$ particles can be formulated using differential forms. Unlike other formulations, this method is easy to use since it yields the Dirac equation in terms of well-known elements such as partial derivatives and special relativistic Dirac matrices.

Here we give a short review of the formalism described in more detail elsewhere [1]. In terms of orthonormal frames (one forms) $\omega^{\mu}$, every 4-dimensional pseudo-Riemannian metric (with signature - 1) can be expressed in the form

$$
d s^{2}=-\left(\omega^{0}\right)^{2}+\left(\omega^{1}\right)^{2}+\left(\omega^{2}\right)^{2}+\left(\omega^{3}\right)^{2}=\eta_{\mu \nu} \omega^{\mu} \otimes \omega^{\nu} .
$$

Here $\eta_{\mu \nu}$ denotes the Minkowski metric. Comparison with the metric for the space-time of interest gives the one-forms $\omega_{v}^{\mu}$ by inspection. From the basis forms $\omega^{\mu}$, the connection one-forms $\omega^{\mu}$ and Ricci rotation coefficients $\gamma_{\alpha \beta}^{\mu}$ can be found from

$$
d \omega^{\mu}=-\omega_{v}^{\mu} \wedge \omega^{v}=\frac{1}{2} b_{\alpha \beta}^{\mu} \omega^{\alpha} \wedge \omega^{\beta},
$$

where $\wedge$ denotes the exterior product and $d$ denotes the exterior derivative. The Ricci rotation coefficients can be found from

$$
\omega_{\mu \nu}=\gamma_{\mu \nu \alpha} \omega^{\alpha}
$$

or from

$$
\gamma_{\alpha \beta \gamma}=-\frac{1}{2}\left(b_{\alpha \beta \gamma}-b_{\gamma \alpha \beta}-b_{\beta \gamma \alpha}\right) \text {. }
$$

The basis vectors $\omega_{\mu}$ dual to the basis forms $\omega^{\mu}$ can be found from

$$
\omega^{\mu} \cdot \omega_{v}=\delta_{v}^{\mu}
$$

In holonomic (coordinate) frames, this becomes the standard relation

$$
d x^{\mu} \cdot e_{v}=d x^{\mu} \cdot \partial_{v}=\omega_{v}\left(x^{\mu}\right)=\delta_{v}^{\mu} .
$$


The coordinate derivative $a_{; \nu}^{\mu}$ of a vector $\omega_{\mu} a^{\mu}$ can be expressed as

$$
a_{; v}^{\mu}=\omega_{v}\left(a^{\mu}\right)+\gamma_{\alpha \nu}^{\mu} a^{\alpha}
$$

where the first term is due to the change in the component $a^{\mu}$ and the second to the change in the basis vectors $\omega_{\mu}$. Except in Cartesian coordinates where the basis vectors are position independent, the position dependent basis vectors usually make a nonvanishing contribution even in flat space, e.g. in spherical coordinates. In spin space associated with the Dirac equation, the basis spinors also are usually position dependent except for Cartesian coordinates in flat space. It is because of this difficulty that most workers write the Dirac equation in Cartesian coordinates and then express it in the desired system. But if the change in spinor basis vectors are accounted for, the Dirac equation can be directly expressed in the desired system. The covariant derivation of a spinor $\psi$ can be expressed as

$$
\nabla_{\mu} \phi=\omega_{\mu}(\psi)-\Gamma_{\mu} \psi
$$

where $\Gamma_{\mu}$ is the spinor connection given by

$$
\Gamma_{\mu}^{g}=-\frac{1}{4} \gamma_{\alpha \beta \mu} \gamma^{\alpha} \gamma^{\beta}+a_{\mu} I=\Gamma_{\mu}+a_{\mu} I
$$

and $a_{\mu}$ is an arbitrary vector [1] which can be related to the vector potential by

$$
a_{\mu} \rightarrow i(\hbar / c) e a_{\mu}
$$

(take $\hbar=1=c$ ). That the quantity $a_{\mu}$ follows directly from Shur's lemma and does not need to be incorporated in an ad hoc manner, is not generally known [1].

In orthonormal Cartan frames, the curved space Dirac equation

$$
\left(\gamma^{\mu} \nabla_{\mu}+m \psi\right)=0
$$

can be expressed as

$$
\gamma^{\mu}\left(\omega_{\mu}(\psi)-\Gamma_{\mu}^{g} \psi\right)+m \psi=0
$$

or as [1] for general $a_{\mu}$ and [6] for $a_{\mu}=0$

$$
\left[\gamma^{\mu}\left(\omega_{\mu}-i e a_{\mu}-\Gamma_{\mu}\right)+m\right] \psi=0 .
$$

This formulation quickly and easily yields the Dirac equation in terms of well-known elements such as partial derivatives and special relativistic Dirac matrices as will be seen below for spherically symmetric spaces.

\section{Spherically Symmetric Spaces}

The metric for spherically symmetric spaces such as for black holes, neutron stars, naked singularities, etc. can be written as

$$
d s^{2}=-A^{2} d t^{2}+B^{2} d t^{2}+r^{2} d \theta^{2}+r^{2} \sin ^{2} \theta d \phi^{2},
$$

where

$$
A^{2}=B^{-2}=1-2 M r^{-1}+Q^{2} r^{-2}
$$


and

$$
\begin{aligned}
G m c^{-2} & =m, \\
G^{1 / 2} Q c^{-2} & =Q .
\end{aligned}
$$

Here $m$ and $Q$ are given in geometrized units where $M=6.8 \times 10^{-56} \mathrm{~cm}$ and $Q=1.4 \times 10^{-34} \mathrm{~cm}$ for the electron.

A convenient set of orthonormal basis forms is

$$
\omega^{0}=A d t, \omega^{1}=B d r, \omega^{2}=r d \theta, \omega^{3}=r \sin \theta d \phi,
$$

while the dual basis vectors are

$$
\omega_{0}=A^{-1} \partial_{t}, \omega_{1}=B^{-1} \partial_{r}, \omega_{2}=r^{-1} \partial_{\theta}, \omega_{3}=(r \sin \theta)^{-1} \partial_{\phi} .
$$

For the basis forms (3.3), the Ricci rotation coefficients are

$$
\gamma_{010}=-A_{r}(A B)^{-1}, \gamma_{212}=\gamma_{313}=(r B)^{-1}, \gamma_{323}=\cot \theta / r \text {, }
$$

while the spinor connection is given by

$$
\begin{aligned}
& \Gamma_{0}=A_{r}(2 A B)^{-1} \gamma^{0} \gamma^{1}+i e a_{0}, \\
& \Gamma_{1}=0+i e a_{1}, \\
& \Gamma_{2}=-(2 r B)^{-1} \gamma^{2} \gamma^{1}+i e a_{2}, \\
& \Gamma_{3}=(2 r B)^{-1} \gamma^{3} \gamma^{1}-(2 r)^{-1} \cot \theta \gamma^{3} \gamma^{2}+i e a_{3} .
\end{aligned}
$$

Exterior to a spherical charge, the vector potential is

$$
a_{0}=q(r A)^{-1} \text {. }
$$

Substituting into (2.3) yields the curved space Dirac equation

$$
\begin{aligned}
& {\left[\gamma^{0} A^{-1}\left(\partial_{t}-i e q r^{-1}\right)+\gamma^{\prime} B^{-1}\left(\partial_{r}+r^{-1}+A_{r}(2 A)^{-1}\right)+\gamma^{2} r^{-1}\left(\partial_{\theta}+\frac{1}{2} \cos \theta\right)\right.} \\
& \left.\quad+\gamma^{3}(r \sin \theta)^{-1} \partial_{\phi}+m\right] \psi=0 .
\end{aligned}
$$

The substitutions

$$
\psi=\chi \sin ^{-1 / 2} \theta
$$

and

$$
i \gamma^{1} K=\gamma^{0}\left(\gamma^{2} \partial_{\theta}+\gamma^{3}(\sin \theta)^{-1} \partial_{\phi}\right),
$$

reduce the Dirac equation to

$$
0=\left[-A^{-1}\left(\partial_{t}-i e q r^{-1}\right)+\gamma^{0} \gamma^{1} B^{-1}\left(\partial_{r}+r^{-1}+A_{r}(2 A)^{-1}+i \gamma^{1} K r^{-1}+\gamma^{0} m\right] \chi\right.
$$

The eigenvalues of $K$ are $k= \pm 1, \pm 2, \ldots,[8]$ and the substitution

$$
\chi=r^{-1} R e^{-i E t}
$$

yields

$$
0=\left[i A^{-1}\left(E+e q r^{-1}\right)+\gamma^{0} \gamma^{1} B^{-1}\left(\partial_{r}+A_{r}(2 A)^{-1}+i \gamma^{1} k r^{-1}+\gamma^{0} m\right] R .\right.
$$


Because (2.12) contains only two matrices $\gamma^{0}$ and $\gamma^{1}$, they can be replaced by $2 \times 2$ matrices $\gamma^{0}=i\left(\begin{array}{cc}1 & 0 \\ 0 & -1\end{array}\right) \gamma^{1}=\left(\begin{array}{ll}0 & 1 \\ 1 & 0\end{array}\right)$ and $R$ by $R=\left(\begin{array}{l}F \\ G\end{array}\right)$ which yields $0=A^{-1}\left(E+e q r^{-1}\right)\left(\begin{array}{l}F \\ G\end{array}\right)+B^{-1}\left(\partial_{r}+A_{r}(2 A)^{-1}\left(\begin{array}{c}G \\ -F\end{array}\right)+k r^{-1}\left(\begin{array}{c}G \\ F\end{array}\right)+m\left(\begin{array}{c}F \\ -G\end{array}\right)=0\right.$.

An alternative choice of orthogonal basis forms is

$$
\begin{aligned}
& \omega^{0}=A d t, \\
& \omega^{1}=d x+r^{-1}(B-1) x d r, \\
& \omega^{2}=d y+r^{-1}(B-1) y d r, \\
& \omega^{3}=d z+r^{-1}(B-1) z d r,
\end{aligned}
$$

where $r B d r=x \omega^{1}+z \omega^{2}+z \omega^{3}$. This basis has the property that it reduces to a Cartesian basis in flat space. The basis vectors dual to the form of (2.14) are

$$
\begin{aligned}
& \omega_{0}=A^{-1} \partial_{t}, \\
& \omega_{1}=\partial_{x}+r^{-1}\left(B^{-1}-1\right) x \partial_{r}, \\
& \omega_{2}=\partial_{y}+r^{-1}\left(B^{-1}-1\right) y \partial_{r}, \\
& \omega_{3}=\partial_{z}+r^{-1}\left(B^{-1}-1\right) z \partial_{r},
\end{aligned}
$$

where $\partial_{r}=r^{-1}\left(x \partial_{x}+y \partial_{y}+z \partial_{z}\right)$.

The Ricci rotation coefficients are given by

$$
\begin{aligned}
& \gamma_{100}=(r A B)^{-1} A_{r} x, \\
& \gamma_{200}=(r A B)^{-1} A_{r} y, \\
& \gamma_{300}=(r A B)^{-1} A_{r} z, \\
& \gamma_{133}=\gamma_{122}=r^{-1}\left(1-B^{-1}\right) y, \\
& \gamma_{211}=\gamma_{233}=r^{-2}\left(1-B^{-1}\right) x, \\
& \gamma_{322}=\gamma_{311}=r^{-2}\left(1-B^{-1}\right) z,
\end{aligned}
$$

and the spin connection takes the form

$$
\begin{aligned}
& \Gamma_{0}=A_{r}(2 r A B)^{-1} \gamma^{0}\left(x \gamma^{1}+y \gamma^{2}+z \gamma^{3}\right)+i e a_{0}, \\
& \Gamma_{1}=\frac{1}{2}\left(1-B^{-1}\right) r^{-2} \gamma^{1}\left(y \gamma^{2}+z \gamma^{3}\right)+i e a_{1}, \\
& \Gamma_{2}=\frac{1}{2}\left(1-B^{-1}\right) r^{-2} \gamma^{2}\left(x \gamma^{1}+x \gamma^{3}\right)+i e a_{2}, \\
& \Gamma_{3}=\frac{1}{2}\left(1-B^{-1}\right) r^{-2} \gamma^{3}\left(x \gamma^{1}+y \gamma^{2}\right)+i e a_{3} .
\end{aligned}
$$

Substituting into (1.13) yields an alternative form for the curved space Dirac equation

$$
\begin{aligned}
& {\left[\gamma^{0} A^{-1}\left(\partial_{t}-i e q r^{-1}\right)+\gamma^{i} \omega_{i}-r^{-2}\left(1-B^{-1}-\frac{1}{2} r A_{r}(A B)^{-1}\right)\right.} \\
& \left.\cdot\left(x \gamma^{1}+y \gamma^{2}+z \gamma^{3}\right)+m\right] \psi=0 .
\end{aligned}
$$




\section{Decomposition into Subspaces of Definite Angular Momentum}

In this section we exploit the rotational symmetry of the equations we are studying. We decompose the Hilbert space $\mathfrak{H}$ of four component wave functions $\psi$ into angular momentum subspaces $\mathfrak{M}_{k m}$ which are invariant under the Hamiltonian associated with the Dirac equation. On $\mathfrak{M}_{k m}$ the Hamiltonian is a $(2 \times 2)$ matrix of first order differential operators.

In the last section we found the Dirac equation in a spherically symmetric gravitational field or a combined gravitational and electric field is given by

$$
\gamma^{0} A^{-1}\left(\partial_{t} \psi+i e \phi \psi\right)+\vec{\gamma} \cdot \vec{\nabla} \psi+(A-1) \gamma_{r} \partial_{r} \psi+\left((A-1) r^{-1}+1 / 2 A_{r}\right) \gamma_{r} \psi+m \psi=0,
$$

where $A_{r}=\partial A / \partial r, \phi=Q / r$ and $\gamma_{r}=r^{-1}\left(x \gamma_{x}+y \gamma_{y}+z \gamma_{z}\right)$.

Multiplying through by a $\gamma^{0}$ we find

$$
\left.i \partial_{r}=H \psi=A(\alpha \cdot \rho+\beta m) \psi+e \phi \psi+A(A-1)\right) \alpha_{r} \rho_{r} \psi-i A\left((A-1) r^{-1}+1 / 2 A_{r}\right) \alpha_{r} \psi
$$

where $\quad p=-i \nabla, p_{r}=-i \partial_{r}, \beta=i \gamma^{0}, \alpha=-\gamma^{0} \gamma, \quad \alpha_{r}=r^{-1}\left(x \alpha_{x}+y \alpha_{y}+z \alpha_{z}\right)$. The matrices $\alpha$ and $\beta$ are hermitian and satisfy the relations $\beta^{2}=I, \beta \alpha+\alpha \beta=0, \alpha_{i} \alpha_{i}$ $+\alpha_{i} \alpha_{i}=2 \delta_{i j} I$. We will be analyzing the self-adjointness and spectrum of $H$. Now, $H$ is a differential operator acting on four component wave functions $\psi$. The inner product on these wave functions is given by

$$
\left(\psi, \psi^{\prime}\right)=\int \sum_{i=1}^{4} \overline{\psi_{i}(x)} \psi_{i}^{\prime}(x) A^{-1} d^{3} x .
$$

Let $\mathfrak{H}$ be the Hilbert space of all four component wave functions with $(\psi, \psi)^{\prime}<\infty$. The fact we are working in curved space does not effect the decomposition of $\mathfrak{H}$ into subspaces of definite angular momentum. The reader can refer to any of the standard tests on the Dirac equation (see e.g. [7], [9]).

The generators of rotation are $\vec{J}=\vec{L}+(1 / 2) \vec{\sigma}$, where $\vec{L}=\vec{r} \times \vec{p}$ and $\vec{\sigma}=i \gamma^{5} \vec{\alpha}$ with $\gamma^{5}=\gamma^{0} \gamma^{1} \gamma^{2} \gamma^{3}$. We introduce the wellknown operator

$$
K=\beta(\vec{\sigma} \cdot \vec{L}+I)
$$

One can check that $K$ commutes with $\alpha_{r}, \partial_{r}, J$ and $H$. We have

$$
K^{2}=\vec{J}^{2}+1 / 4 I \text {. }
$$

The eigenvalues of $K$ are $k= \pm 1, \pm 2, \pm 3, \ldots$ We decompose $\mathfrak{H}$ into subspaces $\mathfrak{M}_{k, m}$ so that for $\psi \in \mathfrak{M}_{k, m}$

$$
J_{z} \psi=m \psi \quad K \psi=k \psi .
$$

Since both $J_{z}$ and $K$ commute with $H, \mathfrak{M}_{k, m}$ is mapped into itself under $H$.

The operator $\vec{\alpha} \cdot \vec{p}$ can be decomposed as the sum of a radial and angular part as follows (see [7], page 158)

$$
\begin{aligned}
\vec{\alpha} \cdot \vec{p} & =\alpha_{r} p_{r}+\frac{i \alpha_{r}}{r} \vec{\alpha} \cdot \vec{L} \\
& =-i \alpha_{r}\left(\partial_{r}+r^{-1}-r^{-1} \beta K\right) .
\end{aligned}
$$


To proceed further with our calculations we will commit ourselves to a representation of the $\gamma$ matrices

$$
\begin{aligned}
\gamma^{0} & =-i\left(\begin{array}{ll}
I & 0 \\
0 & I
\end{array}\right) & \vec{\gamma} & =\left(\begin{array}{ll}
0 & \vec{\tau} \\
\vec{\tau} & 0
\end{array}\right) \\
\beta & =\left(\begin{array}{ll}
I & 0 \\
0 & I
\end{array}\right) & \vec{\alpha} & =i\left(\begin{array}{ll}
0 & \vec{\tau} \\
-\vec{\tau} & 0
\end{array}\right) \\
\gamma^{5} & =\left(\begin{array}{cc}
0 & I \\
-I & 0
\end{array}\right) & \vec{\sigma} & =\left(\begin{array}{ll}
\vec{\tau} & 0 \\
0 & \vec{\tau}
\end{array}\right),
\end{aligned}
$$

where $\vec{\tau}$ are the three Pauli spin matrices

$$
\tau_{x}=\left(\begin{array}{cc}
0 & 1 \\
1 & 0
\end{array}\right) \quad \tau_{y}=\left(\begin{array}{cc}
0 & -i \\
i & 0
\end{array}\right) \quad \tau_{z}=\left(\begin{array}{cc}
1 & 0 \\
0 & -1
\end{array}\right) .
$$

Restricting $H$ to $\mathfrak{M}_{k m}$, we express the wave function $\psi$ in the form

$$
\psi=r^{-1}\left(\begin{array}{cc}
f_{1}(r) & \chi_{k}^{m} \\
f_{2}(r) & \chi_{-k}^{m}
\end{array}\right)
$$

where $\chi_{k}^{m}$ is a two component spinor depending only on the angular variables and satisfying

$$
\begin{aligned}
(\vec{\tau} \cdot \vec{L}+I) \chi_{k}^{m} & =k \chi_{k}^{m}, \\
J_{z} \chi_{k}^{m} & =\left(L_{z}+1 / 2 \tau_{z}\right) \chi_{k}^{m}=m \chi_{k}^{m}, \\
J^{2} \chi_{k}^{m} & =j(j+1) \chi_{k}^{m}=\left(k^{2}-1 / 4\right) \chi_{k}^{m}, \\
\tau_{r} \chi_{k}^{m} & =\chi_{-k}^{m} .
\end{aligned}
$$

The spinors $\chi_{k}^{m}$ are normalized so that

$$
\left(\psi, \psi^{\prime}\right)=\int_{0}^{\infty} A(r)^{-1}\left(\overline{f_{1}(r)} f_{1}^{\prime}(r)+\overline{f_{2}(r)} f_{2}^{\prime}(r)\right) d r .
$$

Then on $\mathfrak{M}_{k, m}$ the Hamiltonian $H$ is given by a $(2 \times 2)$-matrix of first order differential operators

$$
H=\left(\begin{array}{cc}
e \phi+A m & A^{2} \partial_{r}+\frac{1}{2} A A_{r}+k r^{-1} \\
-A^{2} \partial_{r}-\frac{1}{2} A A_{r}+k r^{-1} & e \phi-A m
\end{array}\right),
$$

where $A_{r}=\partial_{r} A$ and $A=\left(1-2 M r^{-1}+Q^{2} r^{-2}\right)^{1 / 2}, \phi=Q / r$.

\section{Case of an Event Horizon, $M>Q$}

When $M>Q$, the function $A(r)=\left(1-2 M r^{-1}+Q^{2} r^{-2}\right)^{1 / 2}$ vanishes at $r_{0}=$ $M+\sqrt{M^{2}-Q^{2}}$. At this radius there is an event horizon. In the reference frame of a distant observer, any particle beginning in the region $R_{0}=\left\{r: r>r_{0}\right\}$ and moving with a velocity not exceeding the velocity of light will stay in the outer region, $R_{0}$, for an eternity. In our analysis of the Dirac equation we will confine 
our attention to the region $R_{0}$. The Hilbert space $\mathfrak{H}$ of wave functions is space of wave functions on $R_{0}$. The inner produce on $\mathfrak{H}$ is restricted to the integral over the region $R_{0}$. Passing to the subspaces $\mathfrak{M}_{k m}$ of $\mathfrak{H}$ the inner product becomes

$$
(f, g)=\int_{r_{0}}^{\infty} A(r)^{-1}\left(\overline{f_{1}(r)} g_{1}(r)+\overline{f_{2}(r)} g_{2}(r)\right) d r .
$$

We will consider $H$ as defined on $D_{0}$ the $C^{\infty}$-functions of compact support in $R_{0}$ (i.e. we consider $f_{i}$ which are infinitely differentiable and there are numbers $a>r_{0}$ and $b>a$ so that if $r \notin[a, b], f_{i}(r)=0$. The numbers $a$ and $b$ may depend on the function $f$.

To analyze $H$ further we make a coordinate transformation. Let $S(t)$ be the solution to the differential equation

$$
\frac{d}{d t} S(t)=A(S(t))^{2}=1-2 M S(t)^{-1}+Q^{2} S(t)^{-2},
$$

and $S(0)=2 r_{0}$. The physical significance of $S(t)$ is that this function gives the $r$ coordinate of a point moving radially outward at the velocity of light. One can find an implicit formula for $S(t)$ but for our purposes all we will need are the facts that

$$
\begin{aligned}
\lim _{t \rightarrow \infty} S(t) t^{-1} & =1, \\
\lim _{t \rightarrow-\infty}\left(S(t)-r_{0}\right)^{-b_{0} t} & =C_{0},
\end{aligned}
$$

where $C_{0}$ is a positive constant and $b_{0}=2 \sqrt{M^{2}-Q^{2}} r_{0}^{-2}$. Roughly speaking $S(t)$ behaves like $t$ for large positive $t$ and approaches $r_{0}$ exponentially for large negative $t$.

Using this function we transform to a new Hilbert spaces $\mathfrak{H}_{1}$. Let $\mathfrak{H}_{1}$ be the Hilbert space of square integrable two component functions on the whole real line. The inner product on $\mathfrak{S}_{1}$ is given by

$$
(f, g)_{1}=\int_{-\infty}^{\infty} \sum_{i=1}^{2} \overline{f_{i}(x)} g_{i}(x) d x
$$

We define a unitary mapping from $\mathfrak{M}_{k m}$ to $\mathfrak{H}_{1}$ by the formula

$$
\begin{aligned}
(U f)_{i}(x) & =f_{i}(S(x)) A(S(x))^{1 / 2} \\
& =f_{i}(S(x))\left(A(S(x))^{-1} \partial S(x) / \partial x\right)^{1 / 2}
\end{aligned}
$$

Note $U$ preserves inner products, i.e., $(U f, U g)_{1}=(f, g)$. Under this transformation we have

$$
U\left(A(r)^{2} \partial r+1 / 2 A A_{r}\right) f_{i}(x)=\frac{d}{d x}(U f)_{i}(x) .
$$

Then the transformed Hamiltonian in $\mathfrak{H}_{1}$ becomes $H_{1}=U H U^{-1}$ given by

$$
H_{1}=\left(\begin{array}{cc}
e \phi(S(x))+A(S(x)) m & d / d x+k A(S(x)) S(x)^{-1} \\
-d / d x+k A(S(x)) S(x)^{-1} & e \phi(S(x))-A(S(x))
\end{array}\right)
$$


The domain $\mathfrak{D}_{1}$ on which $H_{1}$ acts is $\mathfrak{D}_{1}=U \mathfrak{D}_{0}$ which is simply the space of $C^{\infty}$-functions of compact support.

Theorem 4.1. $H$ is essentially self-adjoint on $\mathfrak{D}_{0}$.

Proof. To prove $H$ is essentially self-adjoint it is sufficient to show $H \mid\left(\mathfrak{M}_{k m} \cap \mathfrak{D}_{0}\right)$ is essentially self-adjoint for each $k$ and $m$. Now $H \mid\left(\mathfrak{M}_{k m} \cap \mathfrak{D}_{0}\right)$ is unitarily equivalent on $H_{1}$ restricted to $\mathfrak{D}_{1}$ given by Eq. 4.3.

Note the functions $\phi(S(x))$ and $A(S(x))$ are bounded $C^{\infty}$-functions. (Recall that as $x \rightarrow-\infty S(x) \rightarrow r_{0}$.) Then we can write $H_{1}$ in the form

$$
H_{1}=D_{0}+V, \text { where } D_{0}=\left(\begin{array}{cc}
0 & d / d x \\
-d / d x & 0
\end{array}\right)
$$

and $V$ is a bounded hermitian operator. The operator $D_{0}$ (which is an hermitian matrix times $-i d / d x)$ is well known to be essentially self-adjoint in $\mathfrak{D}_{1}$. Since $H_{1}$ is bounded perturbation of $D_{0}, H_{1}$ is essentially self-adjoint. In fact, $H_{1}^{n}$ is essentially self-adjoint as can be seen in Chernoff's article [2].

Next we investigate the spectrum of $H_{1}$. Note as $x \rightarrow-\infty$ the differential operator $H_{0}$ approaches

$$
H_{-}=\left(\begin{array}{cc}
e \phi\left(r_{0}\right) & d / d x \\
-d / d x & e \phi\left(r_{0}\right)
\end{array}\right) .
$$

One can easily calculate the spectrum of $H_{-}$and one finds $\sigma\left(H_{-}\right)=\mathbb{R}$ the whole real line. From this one would expect the spectrum of the closure of $H_{1}$ to be the whole real line. This we show to be the case.

Theorem 4.2. The spectrum of the closure of $H_{1}$ is the whole real line.

Proof. Suppose $\lambda$ is real and let $w=e \phi\left(r_{0}\right)-\lambda$. Let

$$
f_{n}(x)=\pi^{-1 / 4} n^{-1 / 2} \exp \left(\frac{-(x+n)^{2}}{2 n}+i w x\right)\left(\begin{array}{l}
1 \\
i
\end{array}\right) .
$$

A straightforward computation shows $\left\|f_{n}\right\|=1, f_{n}$ tends weakly to zero as $n \rightarrow \infty$ and $\left\|\left(H_{1}-\lambda\right) f\right\| \rightarrow 0$ as $n \rightarrow \infty$. Hence $\lambda \in \sigma\left(H_{1}\right)$. This completes the proof of the theorem.

We will prove that $H_{1}$ has no eigenvalues. For this we will need the following lemma.

Lemma 4.3. Suppose $\mathfrak{H}$ is a Hilbert space and $V_{t}$ for $t>a$, is a bounded linear operator on $\mathfrak{H}$ so that $V_{t} f$ is continuous in $t$ for each $f \in \mathfrak{H}$. Suppose $f(t)$ is a solution to the differential equation

$$
\frac{d f(t)}{d t}=V_{t} f(t) \text { for } t>a
$$

where the derivative exists in the strong sense. Suppose

$$
\int_{a}^{\infty}\left\|V_{t}\right\| d t=K<\infty
$$


Then the $\lim _{t \rightarrow \infty} f(t)$ exists and if this limit is zero then $f(t)=0$ for all $t$.

Proof. Suppose the hypotheses of the lemma are satisfied. Suppose $f$ is a solution to $(*)$. Then

$$
\begin{aligned}
& \frac{d}{d t}\|f(t)\|=\frac{d}{d t} \sqrt{\|f\|^{2}}=\frac{1}{2\|f\|}\left(\left(V_{t} f, f\right)+\left(f, V_{t} f\right)\right), \\
& \frac{d}{d t}\|f(t)\| \leqq\left\|V_{t}\right\|\|f(t)\| .
\end{aligned}
$$

Note if $f(t)=0$ the above inequality is still valid. Hence

$$
\frac{d}{d t} \ln \|f(t)\| \leqq\left\|V_{t}\right\|
$$

and therefore

$$
\ln \left(\left\|f\left(t_{2}\right)\right\| /\left\|f\left(t_{1}\right)\right\|\right) \leqq \int_{t_{1}}^{t_{2}}\left\|V_{t}\right\| d t \leqq K
$$

Hence for $t, t_{0} \in[a, \infty)$ we have

$$
\left\|f\left(t_{0}\right)\right\| e^{-K} \leqq\|f(t)\| \leqq e^{K}\left\|f\left(t_{0}\right)\right\| .
$$

It follows that if $f(t)$ vanishes for any $t$ in $[a, \infty)$ it vanishes for all $t$. Now we have

$$
\begin{aligned}
\left\|f\left(t_{2}\right)-f\left(t_{1}\right)\right\| & \leqq \int_{t_{1}}^{t_{2}}\left\|V_{t} f(t)\right\| d t \\
& \leqq e^{K}\left\|f\left(t_{0}\right)\right\| \int_{t_{1}}^{t_{2}}\left\|V_{t}\right\| d t .
\end{aligned}
$$

Since the integral above is convergent from $t$ to $\infty$ we have $\left\|f\left(t_{2}\right)-f\left(t_{1}\right)\right\| \rightarrow 0$ as $t_{1}, t_{2} \rightarrow \infty$. Hence $f_{\infty}=\lim _{t \rightarrow \infty} f(t)$ exists and

$$
\left\|f\left(t_{0}\right)\right\| e^{-K} \leqq\left\|f_{\infty}\right\| \leqq e^{K}\left\|f\left(t_{1}\right)\right\| .
$$

Thus if $f_{\infty}=0, f(t)=0$ for all $t$. This completes the proof of the lemma.

Theorem 4.4. $H_{1}$ has no eigenvalues.

Proof. Suppose $\lambda$ is real. We will show $(H-\lambda) f=0$ has no $L^{2}$ solution. To this end suppose $\left(H_{1}-\lambda\right) f=0$. Let $w=e \phi\left(r_{0}\right)-\lambda$ and let

$$
f(x)=\left(\begin{array}{c}
a_{1}(x) e^{i w x}+a_{2}(x) e^{-i w x} \\
i a_{1}(x) e^{i w x}-i a_{2}(x) e^{-i w x}
\end{array}\right) .
$$

A straightforward computation shows that

$$
\frac{d}{d k} a_{i}(x)=\sum_{i=1}^{2} V_{i j}(x) a_{j}(x),
$$


where $\left\{V_{i j}\right\} \rightarrow 0$ exponentially as $x \rightarrow-\infty$. Hence by Lemma 4.3 we have $\lim _{x \rightarrow-\infty} a_{i}(x)=a_{i}(-\infty)$ exists and both $a_{i}(-\infty)$ can not be zero if $f \neq 0$. But from the form of $f(x)$ it is clear that $f$ cannot be square integrable. Hence $H_{1}$ has no eigenvalues. This completes the proof of the theorem.

We have shown that $H_{1}$ has continuous spectrum the whole real line and no point spectrum. We conjecture $H_{1}$ has no singular continuous spectrum.

When we mentioned the result of Theorem 4.4 to Professor Shale he asked if the presence of a black hole prevents the existence of bound states in general. It seems the answer is yes. Suppose we have a static space time manifold with no singularities except for an event horizon around a single black hole. Suppose $\lambda$ is an eigenvalue of the Dirac Hamiltonian and $\psi$ is the associated eigenvector. In general $\psi$ cannot vanish on any open set and so $\psi$ will be non-zero in a neighborhood of the black hole. But Theorem 4.4 indicates that $\psi$ cannot be square integrable near the black hole. It seems the existence of a black hole anywhere in the universe prevents the existence of bound states.

In physical terms one may describe the situation as follows. Imagine a hydrogen atom on Earth and a black hole a light year away. There is a positive probability (admittedly a very small one) that the electron in that hydrogen atom will fall into the black hole. If one makes the very crudest estimates of the probability, $P$, that an electron on Earth will disappear in one second due to a black hole a light year away one finds $P<\exp \left(-10^{20}\right)$. In short the effect is small.

\section{Case of a Naked Singularity, $Q>M$}

For the case of $Q>M$ the function $A(r)$ never vanishes and as $r \rightarrow 0, A(t)$ approaches infinity like $Q r^{-1}$. As in the last section we will make a change of coordinate. Let $S(t)$ be the solution to the differential equation

$$
\frac{d s}{d t}=A(S(t))^{2}=1-2 M S(t)^{-1}+Q^{2} S(t)^{-2}
$$

subject to the additional condition that $S(t) \rightarrow 0$ as $t \rightarrow 0$ from the right. The function $S(t)$ gives the $r$ coordinate of a point moving radially outward at the velocity of light. One can show that

$$
\begin{aligned}
\lim _{t \rightarrow 0^{+}} t^{-1 / 3} S(t) & =\left(3 Q^{2}\right)^{1 / 3} \\
\lim _{t \rightarrow \infty} t^{-1} S(t) & =1 .
\end{aligned}
$$

Let $\mathfrak{H}_{1}$ be the Hilbert space of two component square integrable functions on the positive real axis. The inner product on $\mathfrak{H}_{1}$ is given by

$$
(f, q)_{1}=\int_{0}^{\infty} \sum_{i=1}^{2} \overline{f_{i}(x) q_{i}}(x) d x .
$$

We define a unitary mapping from $\mathfrak{M}_{k m}$ onto $\mathfrak{H}_{1}$ by the formula

$$
\begin{aligned}
(U f)_{i}(x) & =f_{i}(S(x)) A(s(x))^{1 / 2} \\
& =f_{i}(S(x))\left(A(s(x))^{-1} \partial S(x) / \partial x\right)^{1 / 2} .
\end{aligned}
$$


As in the last section $U$ preserves the inner product, i.e. $(U f, U g)_{1}=(f, g)$ and under this transformation $H$ becomes $H_{1}=U H U^{-1}$ given by

$$
\begin{aligned}
H & =\left(\begin{array}{cc}
e \phi(S(x))+A(S(x)) m & d / d x+k A(S(x)) S(x)^{-1} \\
-d / d x+k A(S(x)) S(x)^{-1} & e \phi(S(x))-A(S(x)) m
\end{array}\right), \\
H & =H_{0}+V \\
H_{0} & =\left(\begin{array}{cc}
m & d / d x+u \\
-d / d x+u & -m
\end{array}\right), \quad V=\left(\begin{array}{cc}
v_{1}(x) & 0 \\
0 & v_{2}(x)
\end{array}\right),
\end{aligned}
$$

where $u(x)=k A(S(x)) S(x)^{-1}, \quad v_{1}(x)=e \phi(S(x))+(A(S(x))-1) m$ and $v_{2}(x)=e \phi$ $\cdot(S(x))-(A(S(x))-1) m$. We note $v_{i}(x)$ and $u(x)$ go to zero like $x^{-1}$ for large $x$ and $v_{i}(x)$ goes to infinity like $x^{-1 / 3}$ as $x \rightarrow 0^{+}$and $u(x)$ goes to infinity like $x^{-2 / 3}$ as $x \rightarrow 0^{+}$.

Lemma 5.1. If $f, g \in \mathfrak{D}\left(H_{1}^{*}\right)$ then $f$ and $g$ are continuous and

$$
\langle f, g\rangle_{1}=\left(f, H_{1}^{*} g\right)_{1}-\left(H_{1}^{*} f, g\right)_{1}=f_{1}(0) g_{2}(0)-f_{2}(0) g_{1}(0) \text {. }
$$

Proof. We recall that the definition of the domain of $H$ is such that the domain $\mathfrak{D}\left(H_{1}\right)$ is all $C^{\infty}$-function of compact support in $(0, \infty)$ (i.e. if $F \in \mathfrak{D}\left(H_{1}\right) f_{i}(x)=0$ in a neighborhood of zero and $\infty$ ). From the theory of ordinary differential operators as given in ([4], Chapter XIII) it follows that if $f \in \mathfrak{D}\left(H_{1}^{*}\right)$ and $H_{1}^{*} f=h$, then the $f_{i}(x)$ are absolutely continuous and have derivatives which are in $L^{2}(a, b)$ for $0<\mathrm{a}<\mathrm{b}<\infty$. Integrating the equation $H_{1}^{*} f=h$ we find

$$
\begin{aligned}
& f_{1}(x)=e^{\eta(x)}\left(f_{1}(0)-\int_{0}^{x} e^{-\eta(y)}\left(h_{2}(y)-\left(m-v_{2}(y)\right) f_{2}(y)\right) d y\right) \\
& f_{2}(x)=e^{-\eta(x)}\left(f_{2}(0)+\int_{0}^{x} e^{\eta(y)}\left(h_{1}(y)-\left(m+v_{1}(y)\right) f_{1}(y)\right) d y\right),
\end{aligned}
$$

where $\eta(x)=\int_{0}^{x} u(y) d y$. Since $u(x)$ behaves like $x^{-2 / 3}$ for small $x$ the integrand for $\eta(x)$ is well defined and $\eta(x) \rightarrow 0$ as $x \rightarrow 0$. Since $v_{i}(x)$ is in $L^{2}(0, b)$ for $0<b<\infty$, we have $v_{i}(y) f_{i}(y)$ in $L^{1}(0, b)$ and therefore the expressions for 5.3 are integrable and therefore $f_{i}(x)$ is continuous. In particular we have $\lim _{x \rightarrow 0^{+}} f_{i}(x)=f_{i}(0)$ (i.e. the $f_{i}$ are continuous at zero). From these remarks it follows that if $f, g \in \mathfrak{D}\left(H_{1}^{*}\right)$ then

$$
\begin{aligned}
\langle f, g\rangle_{1} & =\left(f, H_{1}^{*} g\right)_{1}-\left(H_{1}^{*} f, g\right)_{1} \\
& =\lim _{b \rightarrow \infty a \rightarrow 0^{+}} \int_{a}^{b} \sum_{i=1}^{2} f_{i}(x)\left(H_{1}^{*} g\right)_{i}(x)-\left(H_{1}^{*} f\right)_{i}(x) g_{i}(x) d x \\
& =\left.\lim _{b \rightarrow \infty a \rightarrow 0^{+}}\left(-f_{1}(x) f_{2}(x)+f_{2}(x) f_{1}(x)\right)\right|_{\substack{x=b \\
x=a}} \\
& =f_{1}(0) g_{2}(0)-f_{2}(0) g_{1}(0) .
\end{aligned}
$$

This completes the proof of the lemma.

We remark the same result holds for $H_{0}$. 
The bilinear form \langle\rangle$_{1}$ on the domain $\mathfrak{D}\left(H_{1}^{*}\right)$ is directly related to the deficiency spaces of $H_{1}^{*}$ and the possible self-adjoint extensions of $H_{1}$. We refer to [4, Chap. XII, Sect. 4] for a discussion of extensions of hermitian operations. From the form of \langle\rangle$_{1}$ given in (5.2) it is clear that this form is the difference of two positive rank one bilinear forms. Each $f \in \mathfrak{D}\left(H_{1}^{*}\right)$ can be uniquely expressed in the form $f=f_{0}+f_{+}+f_{-}$with $f_{0} \in \mathfrak{D}\left(H_{1}\right), f_{+}, f_{-} \in \mathfrak{D}\left(H_{1}^{*}\right)$ with $H_{1}^{*} f_{ \pm}= \pm i f_{ \pm}$. Then if $f, g \in \mathfrak{D}\left(H_{1}^{*}\right)$ with $f=f_{0}+f_{+}+f_{-}$and $g=g_{0}+g_{+}+g_{-}$then $\langle f, g\rangle_{1}=2 i\left(f_{+}\right.$, $\left.g_{+}\right)_{1}-2 i\left(f_{-}, g_{-}\right)_{1}$. Since \langle\rangle$_{1}$ is the difference of two positive rank one bilinear forms (times $i$ ) it follows that $H_{1}$ has deficiency indices $(1,1)$. Hence the deficiency indices of $H_{1}$ (and $H_{0}$ ) are $(1,1)$. The self-adjoint extensions of $H_{1}$ (or $H_{0}$ ) are obtained by imposing a boundary condition at $x=0$ so as to make \langle\rangle$_{1}$ vanish. It is clear that a necessary and sufficient condition for the vanishing of \langle\rangle$_{1}$ is the requirement that the ratio $f_{1}(0) / f_{2}(0)$ be real. We parametrize the possible boundary condition with a parameter $\theta$ with $0<\theta<\pi$ and the boundary condition $f_{1}(0) \sin \theta+f_{2}(0) \cos \theta=0$. We have shown

Theorem 5.2. $H_{1}$ and $H_{0}$ have deficiency indices $(1,1)$ and have self-adjoint extensions given below.

Definition 5.3 .

$$
\begin{aligned}
& H_{1 \theta}=H_{1}^{*} \mid \mathfrak{D}_{1 \theta}, \text { where } \mathfrak{D}_{1 \theta}=\left\{f \in \mathfrak{D}\left(H_{1}^{*}\right) ; f_{1}(0) \sin \theta+f_{2}(0) \cos \theta=0\right\}, \\
& H_{0 \theta}=H_{0}^{*} \mid \mathfrak{D}_{0 \theta}, \text { where } \mathfrak{D}_{0 \theta}=\left\{f \in \mathfrak{D}\left(H_{0}^{*}\right) ; f_{1}(0) \sin \theta+f_{2}(0) \cos \theta=0\right\} .
\end{aligned}
$$

Now we analyse the spectrum of $H_{0 \theta}$. Let $Q_{m}=(-\infty,-m] \cup[m, \infty)=\mathbb{R}-(-m, m)$ and let $Q_{m}^{0}$ be the interior of $Q_{m}$, i.e. $Q_{m}^{0}=(-\infty,-m) \cup(m, \infty)$. First we will show that the spectrum of $H_{0 \theta}$ contains $Q_{m}$. For $\lambda \in Q_{m}^{0}$ let

$$
f_{n \lambda}(x)=(1 / 2) n^{-3 / 2} x \exp \left(-(2 n)^{-1} x+i \lambda x \sqrt{1-m^{2} \lambda^{-2}}\right)\left(\begin{array}{c}
\sqrt{1+m \lambda^{-1}} \\
-i \sqrt{1-m \lambda^{-1}}
\end{array}\right) .
$$

A straightforward computation shows that $\left(H_{0 \theta}-\lambda\right) f_{n \lambda} \rightarrow 0$ as $n \rightarrow \infty,\left\|f_{n \lambda}\right\|=1$ and $f_{n \lambda}$ tends to zero weakly as $n \rightarrow \infty$. It follows that $\lambda \in \sigma\left(H_{0 \theta}\right)$ the spectrum of $H_{0 \theta}$. Since $\sigma\left(H_{0 \theta}\right)$ is closed we have $Q_{m} \subset \sigma\left(H_{0 \theta}\right)$.

Next we will show that the spectrum of $H_{0 \theta}$ is $Q_{m}$ plus at most a single point in $(-m, m)$ and that point is an eigenvalue of multiplicitly one. To see this assume $f \in \mathfrak{D}\left(H_{0 \theta}\right)$. Then a straightforward computation shows

$$
\begin{aligned}
\left\|H_{0 \theta} f\right\|^{2}= & \int_{0}^{\infty}\left|(-d / d x+u) f_{i}\right|^{2} d x+\int^{\infty}\left|(d / d x+u) f_{2}\right|^{2} d x \\
& +m^{2}\|f\|^{2}+m \sin (2 \theta)\left(\left|f_{1}(0)\right|^{2}+\left|f_{2}(0)\right|^{2}\right) .
\end{aligned}
$$

For $0 \leqq \theta \leqq \pi / 2, \sin 2 \theta \geqq 0$ and all the above terms are positive. Hence for $0 \leqq \theta \leqq \pi / 2$ we have $H_{0 \theta}^{2} \geqq m^{2}$ I and therefore $\sigma\left(H_{0 \theta}\right) \subset Q_{m}$ and since we have the reverse inclusion we have $\sigma\left(H_{0 \theta}\right)=Q_{m}$ for $0 \leqq \theta \leqq \pi / 2$.

For $\theta$ satisfying $\pi / 2<\theta<\pi$ the last term of 5.4 is negative and it is therefore possible that there may be an $f \in \mathfrak{D}\left(H_{0 \theta}\right)$ with $\left\|H_{0 \theta} f\right\|^{2}<m^{2}\|f\|^{2}$. Since the last term of 5.4 is a rank one bilinear form on $\mathfrak{D}\left(H_{0 \theta}\right)$ (recall $f_{1}(0)$ and $f_{2}(0)$ are related 
by a boundary condition) it follows that the spectral projection for $H_{0 \theta}$ for the open interval $(-m, m)$ can be at most a rank one projection. Hence if $H_{0 \theta}$ has any spectrum in $(-m, m)$ it must be a single eigenvalue of multiplicity one. Hence, we have shown

Theorem 5.4. For $0 \leqq \theta \leqq \pi / 2 \sigma\left(H_{0 \theta}\right)=Q_{m} \quad$ and for $\pi / 2<\theta<\pi \quad \sigma\left(H_{0 \theta}\right) \supset Q_{m}$ and any spectrum of $H_{0 \theta}$ in the complement of $Q_{m}$ must be at most a single eigenvalue of multiplicity one.

We briefly discuss the relation between $\theta$ and the eigenvalue $\lambda \varepsilon(-m, m)$. When $u=0$ (see Eq. 5.1) we have

$$
\tan \theta=-\sqrt{\frac{m-\lambda}{m+\lambda}} .
$$

In general the relation between $\lambda$ and $\theta$ depends in a nontrivial fashion on the function $u$. Our calculations indicate that for the functions $u$ occurring in 5.1 all eigenvalues $\lambda$ between $-m$ and $+m$ can occur by a proper choice of $\theta$. However, not every $\theta$ satisfying $\pi / 2<\theta<\pi$ leads to an eigenvalue $\lambda$. For example for $u>0(k>1)$ it appears that as $\lambda$ goes between $-m$ to $+m, \theta$ goes between $\theta_{0}$ and $\pi / 2$ where $\pi<\theta_{0}<\pi / 2$. As $u$ increases ( $k$ increases) $\theta_{0}$ approaches $\pi / 2$.

If $E(\lambda)$ is the spectral resolution of a self-adjoint operator $A$ then the essential spectrum of $A$ is the closed set $\sigma_{e}(A)$ of all real $\lambda$ so that $E(\lambda+\varepsilon)-E(\lambda-\varepsilon)$ is a projection of infinite rank for all $\varepsilon>0$. We will show that $\sigma_{e}\left(H_{1 \theta}\right)=Q_{m}$. It follows that any points of the spectrum of $H_{1 \theta}$ in the open interval $(-m, m)$ must be eigenvalues of finite multiplicity.

We have shown that $\sigma_{e}\left(H_{0 \theta}\right)=Q_{m}$ and we will show $\sigma_{e}\left(H_{1 \theta}\right)=Q_{m}$ by showing $V$ is $H_{0 \theta}$ compact. This means $\mathfrak{D}(V) \supset \mathfrak{D}\left(H_{0 \theta}\right)$ and if $f_{n}$ is a sequence which tends to zero weakly (i.e., $\left(h, f_{n}\right) \rightarrow 0$ as $n \rightarrow \infty$ for all $\left.h \in \mathfrak{H}_{1}\right)$ and $\left\|H_{0 \theta} f_{n}\right\| \leqq K$ for all $n$, where $K$ is a constant, then $\left\|V f_{m}\right\| \rightarrow 0$ as $n \rightarrow \infty$. As shown in Kato's book ([5], Thm. 5.35 page 244) the essential spectrum is unchanged under a relatively compact perturbation, i.e. $H_{0 \theta}$ and $H_{0 \theta}+V=H_{1}$ have the same essential spectrum. We begin with the following lemma.

Lemma 5.5. Let $D_{\theta}$ be the operator

$$
D_{\theta}=\left(\begin{array}{cc}
0 & d / d x \\
-d / d x & 0
\end{array}\right)
$$

on the positive real axis with the boundary condition $f_{1}(0) \sin \theta+f_{2}(0) \cos \theta=0$. Initially $D_{\theta}$ is defined as $C^{\infty}$-two component functions of compact support satisfying the boundary condition at $x=0$ and then we take the closure of this operator. Let $A$ be the operator

$$
A=\left(\begin{array}{ll}
a_{11}(x) & a_{12}(x) \\
a_{21}(x) & a_{22}(x)
\end{array}\right),
$$

where the $a_{i j}$ are functions in $L^{2}([0, b])$ for all $0<b<\infty$ and $a_{i j}(x) \rightarrow 0$ as $x \rightarrow \infty$. Then $A$ is $D_{\theta}$ compact.

Proof. A necessary and sufficient condition for $A$ to be $D_{\theta}$ compact is that 
$A\left(D_{\theta}+i c\right)^{-1}$ is compact for $c>0$. This is what we shall show. Define $\left(A_{n} f\right)_{i}(x)=$ $\sum_{j=1}^{2} a_{i j}(x) f_{j}(x)$ for $0<x<n$ and $\left(A_{n} f\right)_{i}(x)=0$ for $x>n$. A straightforward calculation shows $V_{n}\left(D_{\theta}+i c\right)^{-1}$ is a Hilbert-Schmidt class operator. (An operator $B$ is Hilbert-Schmidt if $B * B$ is of finite trace. It is well known that Hilbert-Schmidt operators are compact.) A sketch of this calculation is as follows. We have

$$
D_{\theta}=U T_{\theta} U^{-1}
$$

where

$$
U=\frac{1}{2}\left(\begin{array}{cc}
1 & -i \\
-i & 1
\end{array}\right) \quad T_{\theta}=\left(\begin{array}{cc}
-i d / d x & 0 \\
0 & i d / d x
\end{array}\right)
$$

where in $T_{\theta}$ the boundary condition $f_{2}(0)=\alpha f_{1}(0)$ with $\alpha=-i e^{-2 i \theta}$ is imposed. The resolvent $T_{\theta}$ is

$$
\begin{aligned}
\left(\left(T_{\theta}+i c\right)^{-1} f\right)_{1}(x) & =\int_{0}^{x} e^{-c(x-y)} f_{1}(y) d y-\alpha e^{-c x} \int_{0}^{\infty} d^{-c y} f_{2}(y) d y, \\
\left(\left(T_{\theta}+i c\right)^{-1} f\right)_{2} & =-\int_{x}^{\infty} e^{c(x-y)} f_{2}(y) d y .
\end{aligned}
$$

Then we have $A_{n}\left(D_{\theta}+i c\right)^{-1}=A_{n} U\left(T_{\theta}+i c\right)^{-1} U^{-1}=U\left(U^{-1} A_{n} U\left(T_{\theta}+i c\right)^{-1} U^{-1}\right.$. Since the Hilbert-Schmidt operators are invariant under unitary transforms it is sufficient to show $U^{-1} A_{n} U\left(T_{\theta}+i c\right)^{-1}$ in Hilbert-Schmidt. Since $U^{-1} A_{n} U$ have the same form as $A_{n}$ (the transformation simply replaces the $a_{i j}$ by linear combinations of $\left.a_{i j}\right)$ it suffices to show $A_{n}\left(T_{\theta}+i c\right)^{-1}$ is compact. But we have an explicit formula for this operator. We have

$$
\left(A_{n}\left(T_{\theta}+i c\right)^{-1} f\right)_{i}(x)=\sum_{j=1}^{2} \int_{0}^{\infty} K_{i j}(x, y) f_{j}(y) d y .
$$

Since the functions $a_{i j}(x)$ are in $L^{2}([0, n])$ it follows the functions $K_{i j}(x, y)$ are in $L^{2}\left([0, \infty]^{2}\right)$. Hence $A_{n}\left(D_{\theta}+i c\right)^{-1}$ is a kernal operator with an $L^{2}$ kernal. Such operators are well known to be Hilbert-Schmidt operators. Since the $a_{i j}(x) \rightarrow 0$ as $x \rightarrow \infty$, we have $\left\|A_{n}-A\right\| \rightarrow 0$ as $n \rightarrow \infty$. Hence $\left\|A_{n}\left(D_{\theta}+i c\right)^{-1}-A\left(D_{\theta}+i c\right)^{-1}\right\| \leqq$ $\left\|A_{n}-A\right\|\left\|\left(D_{\theta}+i c\right)^{-1}\right\|=c^{-1}\left\|A_{n}-A\right\| \rightarrow 0$ as $n \rightarrow \infty$. Hence $A\left(D_{\theta}+i c\right)^{-1}$ is the norm limit of a sequence of compact operators. Since the norm limit of a sequence of compact operators is a compact operator it follows $A\left(D_{\theta}+i c\right)^{-1}$ is compact. This completes the proof of the lemma.

Theorem 5.6 $V$ is $H_{0 \theta}$ compact and therefore the essential spectrum of $H_{1 \theta}=H_{0}+V$ is $Q_{m}=(-\infty, m] \cup[m, \infty)$.

Proof. Let $\eta_{1}(x)$ be the function given by

$$
\begin{array}{ll}
\eta_{1}(x)=\int_{0}^{x} u(y) d y & \text { for } 0 \leqq x \leqq 1, \\
\eta_{1}(x)=\eta_{1}(1) & \text { for } x>1 .
\end{array}
$$


Let

$$
S=\left(\begin{array}{cc}
\exp \left(-\eta_{1}(x)\right) & 0 \\
0 & \exp \left(\eta_{1}(x)\right)
\end{array}\right)
$$

A straightforward computation shows $H_{0 \theta}=S D_{\theta} S+W$ where $W$ is a bonded operator since

$$
\begin{aligned}
S D_{\theta} S & =\left(\begin{array}{cc}
0 & d / d x+u \\
-d / d x+u & 0
\end{array}\right) & & \text { for } 0<x<1, \\
& =\left(\begin{array}{cc}
0 & d / d x \\
-d / d x & 0
\end{array}\right) & & \text { for } x>1,
\end{aligned}
$$

and this expression differs for $H_{0 \theta}$ by a matrix of bounded functions.

Now suppose $f_{n}$ is a sequence so that $\left\|f_{n}\right\| \leqq K$ and $\left\|H_{0 \theta} f_{n}\right\| \leqq K$ for all $n$ (where $K$ is a constant) and $f_{n}$ tends weakly to zero as $n \rightarrow \infty$. We will show $\left\|V f_{n}\right\| \rightarrow 0 n \rightarrow \infty$. We have $\left\|H_{0 \theta} f_{n}\right\|=\left\|\left(S D_{\theta} S+W\right) f_{n}\right\| \leqq K$. Then it follows

$$
\left\|S D_{\theta} S f_{n}\right\| \leqq\left\|H_{0 \theta} f_{n}\right\|+\left\|W f_{n}\right\| \leqq K+\|W\|\left\|f_{n}\right\| \leqq K(1+\|W\|)
$$

Then

$$
\left\|D_{\theta} S f_{n}\right\|=\left\|S^{-1} S D_{\theta} S f_{n}\right\| \leqq\left\|S^{-1}\right\|\left\|S D_{\theta} S f_{n}\right\| \leqq\left\|S^{-1}\right\| K(1+\|W\|) .
$$

Hence $\left\|D_{\theta} S f_{n}\right\|$ is bounded and $S f_{n}$ tends weakly to zero since $S$ is bounded. Note $V S^{-1}$ satisfies the hypothesis of the previous lemma. Therefore $V S^{-1}$ is $D_{\theta}$ compact. Hence, since $S f_{n}$ tends weakly to zero and $\left\|D_{\theta} S f_{n}\right\|$ is bounded we have $\left\|V S^{-1}\left(S f_{n}\right)\right\|=\left\|V f_{n}\right\| \rightarrow 0$ as $n \rightarrow \infty$. Hence, we have shown $V$ is $H_{0 \theta}$ compact. As mentioned earlier since $V$ is $H_{0 \theta}$ compact it follows that $H_{1 \theta}=H_{0 \theta}+V$ and $H_{0}$ have the same essential spectrum. This completes the proof of the theorem.

Next we show that both $H_{0 \theta}$ and $H_{1 \theta}$ have no eigenvalues in the continuum, i.e. if $\lambda^{2}>m^{2}$ then $\lambda$ is not an eigenvalue for $H_{1 \theta}$ or $H_{0 \theta}$.

Theorem 5.7. The eigenvalues of $H_{0 \theta}$ and $H_{1 \theta}$ lie in the closed interval $[-m, m]$.

Proof. Suppose $\lambda$ is real and $|\lambda|>m$. Let

$$
Z(t, x)=\left(\begin{array}{cc}
e \phi(s(x))+A(s(x)) m-\lambda & i t+k A(s(x)) s(x)^{-1} \\
-i t+k A(s(x)) s(x)^{-1} & e \phi(s(x))-A(s(x)) m-\lambda
\end{array}\right),
$$

We have $\operatorname{det}(Z(t, x))+t^{2}=\left(e \phi(s(x))-\lambda^{2}\right)-A(s(x))^{2}\left(m^{2}+k^{2} s(x)^{-2}\right)=\Gamma(x)^{2}$, with $\Gamma(x) \geqq 0$. As $x \rightarrow \infty, s(x) \rightarrow \infty, \phi(s(x)) \rightarrow 0$ and $A(s(x)) \rightarrow 1$ and since $\lambda^{2}>m^{2}$ there is an $a>0$, so that $\Gamma(x)>0$ for $x \geqq a$. We have $\operatorname{det}(Z( \pm \Gamma(x), x))=0$ for $x \geqq a$.

Let $\chi_{ \pm}(x)$ be two component functions satisfying

$$
Z( \pm \Gamma(x), x) \chi_{ \pm}(x)=0
$$

with the normalization conditions

$$
\left|\left(\chi_{ \pm}\right)_{1}^{2}\right|+\left|\left(\chi_{ \pm}\right)_{2}^{2}\right|=1
$$

and $\left(\chi_{ \pm}\right)_{2}>0$. 
Now suppose $\lambda$ is an eigenvalue of $H_{1}$ and $f$ is the associated eigenvector. Then we have $\left(H_{1}-\lambda\right) f=0$. We express $f$ in the form

$$
f(x)=a_{1}(x) \chi_{+}(x) e^{i n(x)}+a_{2}(x) \chi_{-}(x) e^{-i n(x)},
$$

where $\eta(x)=\int_{a}^{x} \Gamma(y) d y$. A straightforward computation shows

$$
\frac{d}{d x} a_{i}(x)=\sum_{j=1}^{2} W_{i j}(x) a_{j}(x),
$$

where the functions $W_{i j}(x)$ tend to zero as $x$ approaches infinity like $x^{-2}$ (i.e. $\left|W_{i j}(x)\right| \leqq k x^{-2}$ for $\left.x>a\right)$. Hence it follows from Lemma 4.3 that $a_{i}(x)$ approaches a limit $a_{i}(\infty)$ as $x \rightarrow \infty$ and furthermore both the numbers $a_{i}(\infty)$ cannot be zero unless $f$ is identically zero for $x>a$. If $\mid\left(\left.f_{1}(x)\right|^{2}+\left|f_{2}(x)\right|^{2}\right.$ approaches a nonzero limit as $x \rightarrow \infty$ then $f$ cannot be in $L^{2}$. But if this limit is zero it follows that $f_{i}(x)=0$ for all $x>a$. And the argument of Lemma 4.3 shows that if both $f_{1}\left(x_{0}\right)=0$ and $f_{2}\left(x_{0}\right)=0$ for some $x_{0}$ with $0<x_{0}<\infty$, then $f_{i}(x)=0$ for all $x$. Hence $f$ cannot be square integrable unless $f=0$. Thus, $\lambda$ can not be an eigenvalue. This completes the proof of the theorem.

Theorem 5.8. For the case of neutrinos $(e=m=0) H_{0 \theta}$ has no eigenvalues unless $\theta=\pi / 2$ and $k>1$ or $\theta=0$ and $k<-1$. For these two cases $H_{0 \theta}$ has an eigenvalue at zero of multiplicity one.

Proof. From the proof of Theorem 5.8 it follows for $m=0$, zero can be the only eigenvalue of $H_{0 \theta}$. If $f$ is an eigenfunction for the eigenvalue zero we have $H_{0 \theta} f=0$ or

$$
\frac{d}{d x} f_{2}(x)=-u(x) f_{2}(x), \quad \frac{d f_{1}}{d x}(x)=u(x) f_{1}(x) .
$$

Both these equations are easily solved, and for $k>1$ one finds $f_{1}$ has no square integrable solution and $f_{2}$ has a square integrable solution. Therefore, for $\theta=\pi / 2$, there is an $f \in \mathfrak{D}\left(H_{0 \theta}\right)$ with $H_{0 \theta} f=0$. For $k<-1$ the roles of $f_{1}$ and $f_{2}$ are reversed and for $\theta=0$ there is an $f \in \mathfrak{D}\left(H_{0 \theta}\right)$ with $H_{0 \theta} f=0$. This completes the proof of the theorem.

In summary we have shown the spectrum of $H_{1 \theta}$ (for $Q>M$ and arbitrary $k+ \pm 1, \pm 2, \ldots$, ) consists of essential spectrum $Q_{m}=(-\infty,-m] \cup[m, \infty)$ plus possible eigenvalue confined in $[-m, m]$. In general there will be eigenvalues in this interval. Their existence can be established by constructing wave functions $f$ so that $\left\|H_{1 \theta} f\right\|^{2}<m^{2}\|f\|^{2}$. We note that rough calculations have led us to conclude that for some values of $\theta$ in the interval $(\pi / 2, \pi)$ one will find a "new" bound state corresponding to the bound state in $H_{0 \theta}$ (see Theorem 5.4). By a "new" bound state we mean all but one of the bound states of $H_{1 \theta}$ will be relatively unaffected by $\theta$ (i.e. they will vary continuously with $\theta$ ) and there will be a bound state that suddenly appears at a $\theta_{1}$ and disappears at a second $\theta_{2}$. A more precise understanding of the situation will require numerical computations.

Finally we conjecture that $H_{0 \theta}$ and $H_{1 \theta}$ have no singular continuous spectrum. 


\section{References}

1. Brill, D. R., Cohen, J. M.: Cartan frames and the general relativistic Dirac Equation. J. Math. Phys. 7, 238 (1966)

2. Chernoff, P. R.: Essential self-adjointness of powers of generators of hyperbolic equations. J. Funct. Anal. 12, 401-414 (1973)

3. Cohen, J. M., Kegeles, L. S. : Electromagnetic fields in curved spaces: A constructive procedure. Phys. Rev. D10, 1070-1084 (1974)

4. Dunford, N., Schwartz, J.: Linear operators, Part II. New York: Interscience Publishers 1963

5. Kato, T.: Perturbation theory for linear operators, New York: Springer 1966

6. Lichnerowitz, A.: Bull. Soc. Math. France 92, 11 (1964)

7. Rose, M. E.: Relativistic electron theory, New York: John Wiley and Sons 1961

8. Schroedinger, E.: Commun. Pontif. Acad. Sci. 2, 321 (1938)

9. Schiff, L.: Quantum mechanics New York: McGraw-Hill 1949

Communicated by B. Simon

Received October 7,1981; in revised form February 1, 1982 